\title{
ON LYAPUNOV MAPPING AND ITS APPLICATIONS*
}

\author{
DAIZHAN $\mathrm{CHENG}^{\dagger}$
}

\begin{abstract}
In this paper we investigate the Lyapunov mapping. Some results about the nature of the Lyapunov mapping are revealed. We first consider the properties of the spectrum and the norm of a Lyapunov mapping with those of its restrictions to the subspaces of symmetric and skew symmetric matrices. Secondly, some sufficient conditions are given for the existence of the common quadratic Lyapunov functions for a set of stable matrices. A norm estimation inequality is presented. Next effort is devoted to an algorithm for constructing a common quadratic Lyapunov function of a pair of matrices. The algorithm is based on norm estimation and several properties of Lyapunov mapping which are related to the numerical computations.
\end{abstract}

Key Words. Lyapunov mapping, system with switching models, spectrum, norm, common quadratic Lyapunov function.

1. Introduction. In recent years there has been much interest in the problem of finding a common Lyapunov function of a set of systems [1-3]. It is closely related to the stability and stabilization of the systems with switching or uncertain models[4-7], $H_{\infty}$ control [8], and hybrid systems[9-10].

A particular attention has been paid to the common quadratic Lyapunov function for a set of linear systems because it has some special properties such as

1. The set of systems which share a common quadratic Lyapunov function is convex. Hence it assures that in a linear or near-linear switching the switching process is also stable.

2. It can be applied to nonlinear systems for local stability near equilibrium states.

The main tool for the approach in this paper is the Lyapunov mapping. We give the definition first. Through the paper we use $M_{n}$ for the vector space of $n \times n$ matrices, use $S_{n}$ and $K_{n}$ for its symmetric and skew-symmetric subspaces respectively.

Definition 1.1. [1-2] 1. Given an $n \times n$ matrix A. The mapping, $L_{A}: M_{n} \rightarrow$ $M_{n}$, defined as

$$
L_{A}(X):=A X+X A^{T}
$$

is called the Lyapunov mapping of $A$.

2. $A$ is anti-stable if $\Re \sigma(A)>0$.

In fact $-A$ is stable.

A positive definite matrix $P$ is called the common quadratic Lyapunov function of two anti-stable matrices $A$ and $B$ if $L_{A}(P)>0$ and $L_{B}(P)>0$. In fact, it means

${ }^{*}$ Received on April 15, 2001, aceepted for publication on July 10, 2001. Supported partly by G59837270, G1998020308 of China and National Key Project.

†Institute of Systems Science, Chinese Academy of Sciences, Beijing 100080, P.R.China. E-mail: dcheng@iss03.iss.ac.cn 
the quadratic form $x^{T} P x$ is a common Lyapunov function of the two linear systems

$$
\dot{x}=-A x, \quad \dot{x}=-B x .
$$

The matrix expression of $L_{A}$ is a matrix $M_{A}$ such that $V\left(L_{A}(X)\right)=M_{A} V(X)$. Then ( $L_{A}$ is used for $M_{A}$ ) [1]

$$
L_{A}=A \otimes I+I \otimes A .
$$

That is

$$
V\left(L_{A}(X)\right)=(A \otimes I+I \otimes A) V(X) .
$$

Let $X \in M_{n} . V: M_{n} \rightarrow R^{n^{2}}$ arranges the elements of $X$ row by row as $V(X)=$ $\left(\begin{array}{llll}x_{11} & x_{12} & \cdots & x_{n n}\end{array}\right)^{T} . V^{-1}$ stands for the inverse mapping of $V$.

We define an $n^{2} \times n^{2}$ square matrix, $W_{[n]}$, in the following way: index the rows by: $(1,1)(1,2) \cdots(1, n) \cdots(n, 1) \cdots(n, n)$ and the columns by: $(1,1)(2,1) \cdots$ $(n, 1) \cdots(1, n) \cdots(n, n)$. The $W_{[n]}=\left(w_{(i, j)(I, J)}\right)$ is defined as

$$
w_{(i, j)(I, J)}= \begin{cases}1, & (i, j)=(I, J) \\ 0, & \text { otherwise. }\end{cases}
$$

We call $W_{n}$ the swap matrix because for a given $n \times n$ matrix $A$

$$
V\left(A^{T}\right)=W_{[n]} V(A), \quad \text { and } \quad V(A)=W_{[n]} V\left(A^{T}\right) .
$$

2. The Spectrum of $L_{A}$. Recall that $S_{n}$ and $K_{n}$ are the symmetric and skewsymmetric subspaces of $M_{n \times n}$. It is easy to see that both $S_{n}$ and $K_{n}$ are invariant subspaces of $L_{A}$.

In the following we use $L_{A}^{S}$ and $L_{A}^{K}$ for the restrictions of $L_{A}$ on $S_{n}$ and $K_{n}$ respectively. The spectrum of $L_{A}^{S}$ and $L_{A}^{K}$ will be determined in this section. To begin with, we consider the spectrum of $L_{A}$.

The following Lemma and Theorem are well known or easily verifiable.

Lemma 2.1. Assume two sets of vectors $X_{i}, i=1, \cdots, s \in R^{n}$ and $Y_{j}, j=$ $1, \cdots, t \in R^{n}$ are both linearly independent. Then $X_{i} \otimes Y_{j}, i=1, \cdots, s, q=1, \cdots t$ are linearly independent.

TheOREM 2.2. Let $\left\{\lambda_{1}, \cdots, \lambda_{n}\right\}$ be eigenvalues of $A$. Then the eigenvalues of $L_{A}^{S}$ are $\left\{\lambda_{i}+\lambda_{j} \mid 1 \leq i \leq j \leq n\right\}$ and the eigenvalues of $L_{A}^{K}$ are $\left\{\lambda_{i}+\lambda_{j} \mid 1 \leq i<j \leq n\right\}$.

As an application, we consider the problem of structure invariance. Let $N$ be an $n$ dimensional manifold and $\omega \in T^{2}(N)$ be a quadratic tensor field. Given a dynamic system

$$
\dot{x}=f(x), \quad x \in N .
$$

$\omega$ is said to be $f$-invariant if along the integral curve, $\phi_{f}^{t}(x)$, of $f$ the $\omega$ is invariant. That is, $\left(\phi_{f}^{t}\right) *(\omega)=\omega$ (Remark: "**"is a supscription). (Refer to [11] for the concepts.) 
This definition is a generalization of the structure invariance of Hamiltonian systems [12], where $\omega$ is required to be a non-singular closed two forms.

Consider a linear dynamic system

$$
\dot{x}=A x, \quad x \in R^{n},
$$

and looking for a quadratic tensor field, $\omega$ with a constant structure matrix, $M_{\omega}$. That is

$$
\omega(x, y)=x^{T} M_{\omega} y, \quad \forall x, y \in R^{n} .
$$

The above result can be used to test whether (2.1) has an invariant structure.

Corollary 2.3. System (2.1) has a symmetric invariant structure, iff there exist eigenvalues, $\lambda_{i}, \lambda_{j} \in \sigma(A)$, such that $\lambda_{i}+\lambda_{j}=0$. A has a skew-symmetric quadratic invariant structure, iff there exist eigenvalues, $\lambda_{i}, \lambda_{j} \in \sigma(A), i \neq j$, such that $\lambda_{i}+\lambda_{j}=0$.

Proof. Since $\phi_{A x}^{t}(x)=\exp (A t) x$. $\omega$ is $A x$ invariant, iff for any $x, y \in R^{n}$

$$
\omega(\exp (A t) x, \exp (A t) y)=\omega(x, y), \quad \forall x, y \in R^{n} .
$$

That is equivalent to

$$
\exp (A t)^{T} M_{\omega} \exp (A t)=M_{\omega} .
$$

Using Taylor expansion, we have, by simply denote $M=M_{\omega}$ and collecting terms,

$$
\left\{\begin{array}{l}
A^{T} M+M A=0 \\
\frac{1}{2} A^{2} T M+A^{T} M A+\frac{1}{2} M A^{2}=0 \\
\cdots \\
\frac{1}{k !} \sum_{i=0}^{k}\left(\begin{array}{c}
k \\
i
\end{array}\right) A^{-(k-i) T} M A^{i} \\
\cdots
\end{array}\right.
$$

We have only to find $M$, which satisfies the first equation of (2.3). Because if $M$ satisfies it, then exchanging $A^{T}$ with $M$, we have

$$
\frac{1}{k !} \sum_{i=0}^{k}\left(\begin{array}{c}
k \\
i
\end{array}\right) A^{-(k-i) T} M A^{i}=(1-1)^{k} M A^{k}=0 .
$$

Now the problem becomes find a solution of

$$
L_{A^{T}}(M)=0 .
$$

The exitance of nonzero $M$ follows from Theorem 2.3. The only thing we would like to show is how to construct $M$. When $A$ has a pair of opposite eigenvalues, then we have $\xi_{i}$ and $\xi_{j}$ as the corresponding eigenvectors. Set $\xi_{i} \otimes \xi_{j}+\xi_{j} \otimes \xi_{i}$. According to Lemma 2.1, it is non-zero and $M=V^{-1}\left(\xi_{i} \otimes \xi_{j}+\xi_{j} \otimes \xi_{i}\right)$ is symmetric. Similarly, $M=V^{-1}\left(\xi_{i} \otimes \xi_{j}-\xi_{j} \otimes \xi_{i}\right)$ is also non-zero and skew-symmetric. 
3. Norm of $L_{A}^{S}$. For estimating stability radius of a stable matrix, $A$, the norm of $L_{A}^{S}$ plays an important role. This section will investigate $\left\|L_{A}^{S}\right\|$.

Proposition 3.1. The norm of $L_{A}$ satisfies

$$
\left\|L_{A}\right\|=\max \left\{\left\|L_{A}^{S}\right\|,\left\|L_{A}^{K}\right\|\right\} .
$$

Proof.

$$
\begin{aligned}
\left\|L_{A}\right\|^{2} & =\sup _{0 \neq M \in M_{n}} \frac{\left\langle L_{A} M, L_{A} M>\right.}{<M, M>} \\
& =\sup _{0 \neq(S, K) \in\left(S_{n}, K_{n}\right)} \frac{<L_{A}(S+K), L_{A}(S+K)>}{<S+K, S+K>} \\
& =\sup _{0 \neq(S, K) \in\left(S_{n}, K_{n}\right)} \frac{<L_{A}^{S} S, L_{A}^{S} S>+<L_{A}^{K} K, L_{A}^{K} K>}{<S, S>+<K, K>} \\
& \leq \max \left\{\left\|L_{A}^{S}\right\|^{2},\left\|L_{A}^{K}\right\|^{2}\right\}
\end{aligned}
$$

The other direction of the inequality is trivial. The conclusion follows.

Recall the definition of the swap matrix $W=W_{[n]}$, the following expression is obvious.

$$
\begin{aligned}
& L_{A}^{S}=\left.L_{A}\left(\frac{I+W}{2}\right)\right|_{S_{n}} . \\
& L_{A}^{K}=\left.L_{A}\left(\frac{I-W}{2}\right)\right|_{K_{n}} .
\end{aligned}
$$

In fact we can prove the following:

Proposition 3.2.

$$
\left\|L_{A}^{S}\right\|=\left\|L_{A}\left(\frac{I+W}{2}\right)\right\|
$$

and

$$
\left\|L_{A}^{K}\right\|=\left\|L_{A}\left(\frac{I-W}{2}\right)\right\|
$$

Proof.

$$
\begin{aligned}
\left\|L_{A}\left(\frac{I+W}{2}\right)\right\| & =\sup _{0 \neq X \in M_{n}} \frac{\left\|L_{A}\left(\frac{I+W}{2}\right) X\right\|}{\|X\|} \\
& \geq \sup _{0 \neq S \in S_{n}} \frac{\left\|L_{A}\left(\frac{I+W}{2}\right) S\right\|}{\|S\|}=\sup _{0 \neq S \in S_{n}} \frac{\left\|L_{A}^{S}\right\|}{\|S\|}=\left\|L_{A}^{S}\right\| .
\end{aligned}
$$

On the other hand, decompose $X$ into symmetric and skew symmetric parts as

$$
X=X_{S}+X_{K}, \quad \text { where } X_{S} \in S_{n}, \quad X_{K} \in K_{n} .
$$


Then

$$
\begin{aligned}
& \left\|L_{A}\left(\frac{I+W}{2}\right)\right\|=\sup _{0 \neq X \in M_{n}} \frac{\left\|L_{A}\left(\frac{I+W}{2}\right)\left(X_{S}+X_{K}\right)\right\|}{\left\|X_{S}+X_{K}\right\|} \\
& =\sup _{0 \neq X \in M_{n}} \frac{\left\|L_{A} X_{S}\right\|}{\left\|X_{S}+X_{K}\right\|} \leq \sup _{0 \neq X \in M_{n}} \frac{\left\|L_{A} X_{S}\right\|}{\left\|X_{S}\right\|} \\
& =\sup _{0 \neq X_{S} \in S_{n}} \frac{\left\|L_{A} X_{S}\right\|}{\left\|X_{S}\right\|}=\left\|L_{A}^{S}\right\| .
\end{aligned}
$$

Both inequalities imply (3.4). Equation (3.5) can be proved in a similar way.

Next, we consider a special case when $A$ is normal.

Proposition 3.3. Assume $A$ is normal. Then

1. All $L_{A}, L_{A}\left(\frac{I+W}{2}\right)$ and $L_{A}\left(\frac{I-W}{2}\right)$ are normal.

2. The norm of $L_{A}$ (or $\left.L_{A}^{S}\right)$ is $2 \max \{|\lambda| \mid \lambda \in \sigma(A)\}$.

3. If in addition, $\Re \sigma(A)<0$ (or $\Re \sigma(A)>0$ ), then $\left\|\left(L_{A}\right)^{-1}\right\|=\left\|\left(L_{A}^{S}\right)^{-1}\right\|$.

Proof. 1. Since $A A^{T}=A^{T} A$, then

$$
\begin{aligned}
& (A \otimes I+I \otimes A)^{T}(A \otimes I+I \otimes A)=\left(A^{T} \otimes I+I \otimes A^{T}\right)(A \otimes I+I \otimes A) \\
& \quad=A^{T} A \otimes I+A^{T} \otimes A+A \otimes A^{T}+I \otimes A^{T} A \\
& \quad=A A^{T} \otimes I+A^{T} \otimes A+A \otimes A^{T}+I \otimes A A^{T} \\
& \quad=(A \otimes I+I \times A)(A \otimes I+I \otimes A)^{T},
\end{aligned}
$$

$L_{A}$ is normal.

It is easy to show that for any $A$

$$
L_{A}\left(\frac{I+W}{2}\right)=\left(\frac{I+W}{2}\right) L_{A} .
$$

Using this fact and (3.11), we have

$$
\begin{aligned}
& \frac{(I+W)^{T}}{2}(A \otimes I+I \otimes A)^{T}(A \otimes I+I \otimes A) \frac{I+W}{2} \\
& =\frac{I+W^{-1}}{2}\left(A^{T} \otimes I+I \otimes A^{T}\right)(A \otimes I+I \otimes A) \frac{I+W}{2} \\
& =\frac{I+W^{-1}}{2}\left(A^{T} A \otimes I+I \otimes A^{T} A+A \otimes A+A \otimes A^{T}\right) \frac{I+W}{2} \\
& =\frac{I+W^{-1}}{2}(A \otimes I+I \times A)\left(A^{T} \otimes I+I \times A^{T}\right) \frac{I+W}{2} \\
& =(A \otimes I+I \otimes A) \frac{I+W^{-1}}{2} \frac{I+W}{2}\left(A^{T} \otimes I+I \otimes A^{T}\right) \\
& =(A \otimes I+I \otimes A) \frac{I+W}{2} \frac{I+W^{-1}}{2}\left(A^{T} \otimes I+I \otimes A^{T}\right) \\
& =(A \otimes I+I \otimes A) \frac{I+W}{2} \frac{(I+W)^{T}}{2}\left(A^{T} \otimes I+I \otimes A^{T}\right) .
\end{aligned}
$$

Hence $L_{A}\left(\frac{I+W}{2}\right)$ is normal. Similarly, $L_{A}\left(\frac{I-W}{2}\right)$ is normal.

2. Since $L_{A}$ is normal, $\left\|L_{A}\right\|=\max \left\{|r| \mid r \in \sigma\left(L_{A}\right)\right\}=\max \left\{\left|\lambda_{i}+\lambda_{j}\right| \mid \lambda_{i}, \lambda_{j} \in\right.$ $\sigma(A)\}$. According to Theorem 2.2, $\lambda_{i}+\lambda_{j} \in \sigma\left(L_{A}^{S}\right)$. Hence $\left\|L_{A}^{S}\right\| \geq \max \{|r| \mid r \epsilon$ $\left.\sigma\left(L_{A}^{S}\right)\right\}=\max \left\{\left|\lambda_{i}+\lambda_{j}\right| \mid \lambda_{i}, \lambda_{j} \in \sigma(A)\right\}$. But $\left\|L_{A}^{S}\right\| \leq\left\|L_{A}\right\|$. Hence $\left\|L_{A}^{S}\right\|=\left\|L_{A}\right\|$.

3. Note that the additional condition of stable (or anti-stable) assures the invertibility of $L_{A}$. Now since $S_{n}$ is $L_{A}$ invariant, it is easy to see that

$$
\left(L_{A}^{S}\right)^{-1}=\left.\left(L_{A}\right)^{-1}\right|_{S} .
$$


Similar argument as in 2 shows that

$$
\left\|\left(L_{A}\right)^{-1}\right\|=\left\|\left(L_{A}^{S}\right)^{-1}\right\|=\max \left\{\frac{1}{\left|\lambda_{i}+\lambda_{j}\right|} \mid \lambda_{i}, \lambda_{j} \in \sigma(A)\right\} .
$$

When $n=2$, assume $\sigma(A)=\left\{\lambda_{1}, \lambda_{2}\right\}$. Using Theorem 2.2, since $\operatorname{dim}\left(K_{2}\right)=1$, $\left\|K_{2}\right\|=\left|\lambda_{1}+\lambda_{2}\right|$. But for any matrix $M,\|M\| \geq \max \left\{\left|\lambda_{i}\right| \mid \lambda_{i} \in \sigma(M)\right\}$. Theorem 2.2 implies that $\left\|L_{A}^{S}\right\| \geq\left\|L_{A}^{K}\right\|$. So, $\left\|L_{A}\right\|=\left\|L_{A}^{S}\right\|$.

Our conjecture is $\left\|L_{A}\right\|=\left\|L_{A}^{S}\right\|$.

4. Common Lyapunov Functions for Two Matrices. Based on the technique used in [1], this section will give conditions under which two matrices share a common quadratic Lyapunov function.

Denote by $P_{n}$ the set of positive semi-definite matrices, and its interior, $\operatorname{int}\left(P_{n}\right)$, the set of positive definite matrices. Let $A_{i}, \quad i=1, \cdots, k$ be a set of finite square matrices. $A_{i}, \quad i=1, \cdots, k$ share a common quadratic Lyapunov function if there exists a $P \in \operatorname{int}\left(P_{n}\right)$ such that

$$
A_{i} P+P A_{i}^{T} \in \operatorname{int}\left(P_{n}\right), \quad i=1, \cdots, k
$$

The dual equivalent statement [13] says that (4.1) is equivalent to:

$$
\sum_{i=1}^{k}\left(A_{i}^{T} Y_{i}+Y_{i} A_{i}\right) \in-P_{n}
$$

has no nonzero solution $\left(Y_{1}, \cdots, Y_{k}\right) \in\left(P_{n}\right)^{k}$.

For a notational convenience, we consider when a set of anti-stable matrices $A_{i}$ satisfy (4.1). It is obvious that this statement is equivalent to a set of stable matrices $B_{i}=-A_{i}$ to share a common quadratic Lyapunov function. Precisely, let

$$
\dot{x}=B_{i} x, \quad i=1, \cdots, k .
$$

Set a quadratic Lyapunov function $V=x^{T} P x$. Then (4.1) assures that

$$
\left.\dot{V}\right|_{i}=B_{i} P+P B_{i}^{T}<0, \quad i=1, \cdots, k
$$

So $V$ is a common quadratic Lyapunov function for all $k$ linear dynamic systems.

The following lemma and two convenient sufficient conditions for them to share a common quadratic Lyapunov function are presented in [1] as:

Lemma 4.1. Let $L$ be a linear mapping on $S_{n}$. If

$$
L^{T}+L>0
$$

then there exists a $Q \in \operatorname{int}\left(P_{n}\right)$ such that $L(Q) \in \operatorname{int}\left(P_{n}\right)$.

Then the following result is obtained as 
Theorem 4.2. [1] Assume either $A$ or $B$ is anti-stable, and one of the following two conditions is satisfied, then $A$ and $B$ share a common quadratic Lyapunov function.

$$
\left(L_{A}\right)^{T} L_{B}+\left(L_{B}\right)^{T} L_{A}>0
$$

$$
\left(L_{A}\right)^{T}\left(L_{B}\right)^{T} L_{A} L_{B}+\left(L_{B}\right)^{T}\left(L_{A}\right)^{T} L_{B} L_{A}>0 .
$$

Based on Lemma 4.1 , these two conditions in Theorem 4.2 can be generalized as

Theorem 4.3. Let $A, B$ be as in Theorem 4.2. They share a common quadratic Lyapunov function if there exists a linear mapping $G: S_{n} \rightarrow S_{n}$, which is invertible and $G^{-1}: \operatorname{int}\left(P_{n}\right) \rightarrow \operatorname{int}\left(P_{n}\right)$, such that

$$
\left(L_{A}\right)^{T} G L_{B}+\left(L_{B}\right)^{T} G^{T} L_{A}>0
$$

Particularly, a class of such $G$ has the form $G=\prod_{i=1}^{k} L_{S_{i}}$, where $S_{i}, i=1, \cdots, k$, are anti-stable matrices. Set $S_{i}=I_{n},(4.4)$ follows. Set $S_{1}=B^{T}, S_{2}=A,(4.5)$ follows.

If we set $S_{1}=\left(A^{-1}\right)^{T}, S_{2}=B^{T}$, we get a new condition as

$$
\left(L_{A}\right)^{T}\left(L_{A^{-1}}\right)^{T}\left(L_{B}\right)^{T} L_{B}+\left(L_{B}\right)^{T} L_{B} L_{A^{-1}} L_{A}>0
$$

Example 4.4. Consider two matrices

$$
A=\left(\begin{array}{cc}
1 & 0.3 \\
0.3 & 1
\end{array}\right) \quad \text { and } \quad B=\left(\begin{array}{cc}
0.7 & -1 \\
1 & -0.3
\end{array}\right) .
$$

It is easy to check that $A$ and $B$ satisfy neither (4.4) nor (4.5), but they do satisfy (4.7). Hence, they have common quadratic Lyapunov function.

Next, we try to unify the test conditions in [1] with those in [2]. Let $\pi\left(P_{n}\right)$ be the set of linear mappings: $P_{n} \rightarrow P_{n}$, and $\pi\left(\operatorname{int}\left(P_{n}\right)\right)$ be the set of linear mappings: $\operatorname{int}\left(P_{n}\right) \rightarrow \operatorname{int}\left(P_{n}\right)$.

Two more conditions for anti-stable matrices $A$ and $B$ to share a common quadratic Lyapunov function are given as

THEOREM 4.5. [2] If one of $A$ and $B$ is anti-stable, and there exists a mapping $G>0, G^{-1} \in \pi\left(P_{n}\right)$ and one of the following holds:

$$
\begin{gathered}
\left(L_{A}\right)^{T} G L_{B}+\left(L_{B}\right)^{T} G L_{A}>0, \\
\left(L_{A}\right)^{T}\left(L_{B}\right)^{T} G L_{A} L_{B}+\left(L_{B}\right)^{T}\left(L_{A}\right)^{T} G\left(L_{A} L_{B}\right)>0 .
\end{gathered}
$$


This result can be improved. We need the following lemma.

Lemma 4.6. Assume a linear mapping $G: S_{n} \rightarrow S_{n}$ is nonsingular. Then $G \in \pi\left(P_{n}\right)$, iff, $G \in \pi\left(\operatorname{int}\left(P_{n}\right)\right)$.

Proof. Let $G \in \pi\left(\operatorname{int}\left(P_{n}\right)\right)$. Since $P_{n}$ is a closed subset of $M_{n}$, which is a complete metric space with standard metric inherited from $R^{n^{2}}$ and any linear operator over a finite dimensional normed space is continuous then by the continuity of $G$ it is easy to see that $G\left(P_{n}\right) \subset\left(P_{n}\right)$, i.e., $G \in \pi\left(P_{n}\right)$.

Conversely, if $G \in \pi\left(P_{n}\right)$, we have only to show that for any $p \in \operatorname{int}\left(P_{n}\right), G(p) \in$ $\operatorname{int}\left(P_{n}\right)$. If not, then $G(p)$ should be on the boundary, $B$, of $\operatorname{int}\left(P_{n}\right)$. Since $\operatorname{int}\left(P_{n}\right)$ is an open set in $S_{n}$, there exists an open neighborhood, $p \in V \subset \operatorname{int}\left(P_{n}\right)$. Since $G$ is non-singular, it is a local diffeomorphism. That is, $G(V) \ni G(p)$ is an open set in $S_{n}$. But $G(p)$ is on the boundary. Note that under the subspace topology $S_{n} \subset R^{n \times n}$, the boundary is $B=P_{n} \backslash \operatorname{int}\left(P_{n}\right)$. Hence $G(p) \in B$ implies that $G(V)$ contains points which are not in $P_{n}$. This is a contradiction.

Using Lemma 4.6, and Theorem 4.5, the following is an immediate consequence.

TheOREM 4.7. Assume the linear mapping $G: S_{n} \rightarrow S_{n}$ is non-singular and $G^{-1} \in \pi\left(P_{n}\right)$, or equivalently $G^{-1} \in \pi\left(\operatorname{int}\left(P_{n}\right)\right)$. Moreover

$$
\left(L_{A}\right)^{T} G L_{B}+\left(L_{B}\right)^{T} G^{T} L_{A}>0
$$

where $A$ or $B$ is anti-stable. Then $A$ and $B$ share a common quadratic Lyapunov function.

According to Theorem 4.7, the condition $G>0$ in Theorem 4.5 can be relaxed by " $G$ is nonsingular". So Theorem 4.7 implies Theorems 4.2 and 4.5 .

It is interesting that Theorem 4.7 has a dual result as

Theorem 4.8. Assume that $G \in \pi\left(P_{n}\right)$ is linear and that

$$
\left(L_{A}\right)^{T} G L_{B}+\left(L_{B}\right)^{T} G^{T} L_{A} \leq 0
$$

where either $A$ or $B$ is anti-stable. Then $A$ and $B$ share no common quadratic Lyapunov function.

Proof. Suppose a common quadratic Lyapunov function, $x^{T} P x$, exists. Then

$$
\begin{aligned}
& V^{T}(P)\left(\left(L_{A}\right)^{T} G L_{B}+\left(L_{B}\right)^{T} G^{T} L_{A}\right) V(P)= \\
& \left\langle L_{A}(P), G\left(L_{B}(P)\right)\right\rangle+\left\langle G\left(L_{B}(P)\right), L_{A}(P)\right\rangle>0,
\end{aligned}
$$

a contradiction.

Corresponding to the positive statements of equations (4.4), (4.5) and (4.7), we have the following:

Corollary 4.9. Assume $A$ and $B$ are anti-stable. They share no common quadratic Lyapunov function if one of the following holds:

$$
\left(L_{A}\right)^{T} L_{B}+\left(L_{B}\right)^{T} L_{A} \leq 0
$$




$$
\begin{aligned}
& \left(L_{A}\right)^{T}\left(L_{B}\right)^{-T}\left(L_{A}\right)^{-1} L_{B}+\left(L_{B}\right)^{T}\left(L_{A}\right)^{-T}\left(L_{B}\right)^{-1} L_{A} \leq 0 \\
& \left.\left(L_{A}\right)^{T}\left(L_{A}\right)^{-T}\right)\left(L_{B}\right)^{-T} L_{B}+\left(L_{B}\right)^{T}\left(L_{B}\right)^{-1}\left(L_{A}\right)^{-T} L_{A} \leq 0 .
\end{aligned}
$$

Same argument as used in the proof of Theorem 4.8 can be used to prove the Corollary 4.9 .

5. A Common Lyapunov Function for a Set of Matrices. Let $\left\{A_{1} \cdots A_{k}\right\}$ be a set of anti-stable matrices. We give a necessary and sufficient condition for them to have a common quadratic Lyapunov function.

Proposition 5.1. The set of matrices $\left\{A_{1} \cdots A_{k}\right\}$ have a common quadratic Lyapunov function if and only if the following matrix inequalities

$$
\left[I+L_{D_{i}}\left(L_{C}\right)^{-1}\right](S)>0, \quad i=1, \cdots k-1
$$

have a positive solution $S>0$. Where $C=A_{k}$, and $D_{i}=A_{i}-C$.

Proof. (Sufficiency) Since $L_{A}(X)$ is linear with respect to $A,(5.1)$ can be re-written as

$$
\left[L_{C+D_{i}}\left(L_{C}\right)^{-1}\right](S)=\left[L_{A_{i}}\left(L_{C}\right)^{-1}\right](S)>0 .
$$

It is easy to see that $\left(L_{C}\right)^{-1}(S)$ is the common quadratic Lyapunov function.

(Necessity) Assume the common quadratic Lyapunov function is $X^{t} P X$. Then $S=$ $L_{C}(P)$ is a required solution.

Note that $C$ can be any matrix in the set. Using the Lyapunov mapping, the above result becomes simple. In fact, some early results, e.g., some sufficient conditions in [6-7], can be obtained by setting $S=I$.

Proposition 5.1 cannot provide a common quadratic Lyapunov function directly. There are two problems, which have to be solved. First of all, how to choose $C$. Secondly, how to find a suitable $S$ ? The second is more critical.

It is easy to see that the set of positive definite matrices is an open convex cone in $S_{n}$. Therefore, we hope that the norm of the shifting term, operator $L_{D_{i}}\left(L_{C}\right)^{-1}$, can be as "small" as possible. In such a way, the image of $S$ can be as close to $S$ as possible, and hence remain in the cone.

We give an estimation of the norm of $L_{C}$.

Proposition 5.2.

$$
\sqrt{2}\|C\|_{2} \leq\left\|L_{C}\right\| \leq 2\|C\|_{2},
$$

where the norm $\|\cdot\|_{2}$ stands for the norm of linear operators on $R^{n}$.

Proof. The right half is obvious because

$$
\left\|L_{C} X\right\|=\left\|C X+X C^{T}\right\| \leq 2\|C\|_{2}\|X\| .
$$


As for the left half of (5.2) let $r=\|C\|_{2}$. Then there exists a $\xi$, with $\|\xi\|=1$ such that $C^{T} C \xi=r^{2} \xi$. Since $V^{-1}(\xi \otimes \xi)=\xi \xi^{T}$ and $\left\|\xi \xi^{T}\right\|=1$ we have

$$
\begin{aligned}
\left\|L_{C}\right\|^{2} \geq & \left\|L_{C}\left(\xi \xi^{T}\right)\right\|^{2} \\
= & (\xi \otimes \xi)^{T}\left(C^{T} \otimes I+I \otimes C^{T}\right)(C \otimes I+I \otimes C)(\xi \otimes \xi) \\
= & (\xi \otimes \xi)^{T}\left(C^{T} C \otimes I+I \otimes C^{T} C\right)(\xi \otimes \xi) \\
& +(\xi \otimes \xi)^{T}\left(C^{T} \otimes C+C \otimes C^{T}\right)(\xi \otimes \xi) .
\end{aligned}
$$

By definition of $\xi$, the first term is $2 r^{2}$. Now we have only to show that the second term is non-negative. In fact it is because

$$
\begin{aligned}
& (\xi \otimes \xi)^{T}\left(C^{T} \otimes C+C \otimes C^{T}\right)(\xi \otimes \xi) \\
& =\left(\xi^{T} C^{T} \xi\right) \otimes\left(\xi^{T} C \xi\right)+\left(\xi^{T} C \xi\right) \otimes\left(\xi^{T} C^{T} \xi\right)=\left(\xi^{T} C \xi\right)^{2} \geq 0 .
\end{aligned}
$$

In general, (5.2) is sharp. Consider

$$
A=\left(\begin{array}{ll}
0 & 1 \\
0 & 0
\end{array}\right) .
$$

It is easy to verify that for this $A$ the left half of (5.2) becomes an equality.

Motivated by (5.2), we propose the following way to select $C$ : Choose $C \in$ $\left\{A_{i}, \mid i=1, \cdots, k\right\}$ with largest norm $\|C\|_{2}$. Then

$$
\begin{aligned}
\left\|\left(L_{C}\right)^{-1}\right\| & =\sup _{X \neq 0} \frac{\left\|\left(L_{C}\right)^{-1}(X)\right\|}{\|X\|}=\sup _{X \neq 0} \frac{1}{\frac{\left\|L_{C}(X)\right\|}{\|X\|}} \\
& =\frac{1}{\inf _{X \neq 0} \frac{\left\|L_{C}(X)\right\|}{\|X\|} \leq \frac{1}{\sqrt{2}\|C\|} .}
\end{aligned}
$$

In the next section an algorithm will be developed to search $S$.

6. An Algorithm. Now we consider the problem of searching $S$ with a fixed $C$. First of all, we consider the case of two matrices. This is the most important case because in most cases the switchings occur between two models. Given two anti-stable matrices $A$ and $B$, say, we choose $C=B$ and $D=A-C$. Then consider the mapping

$$
F(S):=S+L_{D}\left(L_{C}\right)^{-1}(S) .
$$

Since $F$ is a linear mapping, we denote it by a matrix $F$. The rows of $F$ are indexed by a set of double indices

$$
\Lambda=\{(i j) \mid 1 \leq i, j \leq n\}
$$

in the order of $(11,21, \ldots, n 1, \ldots, 1 n, \ldots, n n)$ (Remark: the notation above is not defined.).

Let $s=V(S)$ be the row stacking form of $S$, and $T=F S$ with the row stacking form $t=V(T)$. Then we can express (6.1) as

$$
t=\left(\begin{array}{c}
f_{11} \\
\cdots \\
f_{n n}
\end{array}\right)
$$


Equivalently, we have

$$
t_{i j}=f_{i j} s, \quad i, j=1, \cdots, n .
$$

Now $F$ is a given matrix. We are looking for an $S>0$ such that $T=F(S)>0$. Denote by $T^{k}(S)$ the $k$-th principal minor of $T$. A necessary condition is the following.

Lemma 6.1. $T=F(S)$ cannot be positive definite for any $S$ if for some $1 \leq k \leq n$

$$
\operatorname{det}\left(T^{k}(S)\right)=0, \quad \text { for all } S .
$$

Equivalently, the following equation holds:

$$
\sum_{\sigma \in \mathbf{S}_{\mathbf{k}}} \operatorname{det}\left(\begin{array}{llll}
f_{11}^{\lambda_{\sigma(1)}} & f_{12}^{\lambda_{\sigma(2)}} & \cdots & f_{1 k}^{\lambda_{\sigma(k)}} \\
f_{21}^{\lambda_{\sigma(1)}} & f_{22}^{\lambda_{\sigma(2)}} & \cdots & f_{2 k}^{\lambda_{\sigma(k)}} \\
& & \cdots & \\
f_{k 1}^{\lambda_{\sigma(1)}} & f_{k 2}^{\lambda_{\sigma(2)}} & \cdots & f_{k k}^{\lambda_{\sigma(k)}}
\end{array}\right)=0
$$

for all distinct $\lambda_{1}, \lambda_{2}, \cdots, \lambda_{k} \in \Lambda$, where $f_{i j}^{\lambda_{i}}$ is the $\lambda_{i}$-th component of $f_{i j}, \mathbf{S}_{\mathbf{k}}$ is the $k$-th symmetric group, i.e., $\sigma$ is a permutation for $k$ indices $\lambda_{1} \cdots, \lambda_{k}$.

Proof. (6.3) is obvious. Denote

$$
c_{k}(s)=\operatorname{det}\left(T^{k}(S)\right), \quad k=1, \cdots, n
$$

Then

$$
c_{k}(s)=\operatorname{det}\left(\begin{array}{cccc}
\sum_{i, j=1}^{n} f_{11}^{i j} s_{i j} & \sum_{i, j=1}^{n} f_{12}^{i j} s_{i j} & \cdots & \sum_{i, j=1}^{n} f_{1 k}^{i j} s_{i j} \\
\sum_{i, j=1}^{n} f_{21}^{i j} s_{i j} & \sum_{i, j=1}^{n} f_{12}^{i j} s_{i j} & \cdots & \sum_{i, j=1}^{n} f_{2 k}^{i j} s_{i j} \\
\sum_{i, j=1}^{n} f_{k 1}^{i j} s_{i j} & \sum_{i, j=1}^{n} f_{12}^{i j} s_{i j} & \cdots & \sum_{i, j=1}^{n} f_{k k}^{i j} s_{i j}
\end{array}\right) .
$$

It is clear that $c_{k}(S)$ is a $k$-th homogeneous polynomial of $S$. Hence $c_{k}(S) \equiv 0$ if and only if

$$
\frac{\partial^{k} c_{k}}{\partial s_{\lambda_{1}} \cdots \partial s_{\lambda_{k}}}=0, \text { for all } \lambda_{1}, \lambda_{2}, \cdots, \lambda_{k} \in \Lambda
$$

Since each column of $T^{k}(S)$ is linear in $s$, for a chosen set of $\lambda_{1}, \lambda_{2}, \cdots, \lambda_{k} \in \Lambda$ to get the above differentiation, we can only differentiate each column with distinct $\lambda_{j}, j=1,2, \cdots, k$, which yields (6.4).

In general, (6.3) is not verifiable. Unlike (6.3), (6.4) is easily verifiable by computer.

As a necessary condition, we can assume that $F$ satisfies the following

A1: For any $k$, equation (6.4) is not identical to zero. 
Denote by $G_{i j}^{k}$ the co-factor of $t_{i j}$ in $T^{k}(S)$. Observe the following two facts: 1 . since $c_{k}(S)=\sum_{j=1}^{k} t_{i j} G_{i j}^{k}$ and $G_{i j}^{k}, \forall j$ are independent of $t_{i, j}$, we have

$$
\frac{\partial c_{k}}{\partial t_{i j}}=G_{i j}^{k}
$$

2. Since $t_{i j}$ is a linear function of $s$,

$$
\nabla_{s} t_{i j}=\left(f_{i j}\right)^{T}
$$

These two facts yield that the gradient of $c_{k}(s)$ is

$$
\nabla c_{k}(s)=\sum_{i, j=1}^{k} \frac{\partial c_{k}(s)}{\partial t_{i j}} \nabla t_{i j}=\sum_{i, j=1}^{k} G_{i j}^{k}\left(f_{i j}\right)^{T} .
$$

We propose an algorithm for searching $S$. Choose, say $S_{0}=I$. Then at $i$-th step, if for $s_{i}$ all $c_{k}\left(s_{i}\right)>0, k=1, \cdots, n$, we are done. Otherwise, we search a feasible direction $X_{i}$ such that for all $k$ with $c_{k}(s) \leq 0$, they will increase in the direction of $X_{i}$ (for $s$ near $s_{i}$ ), and then set $s_{i+1}=s_{i}+L X_{i}$, where $L>0$ is a suitable step-length.

Let's see how to find $X$ at point $S$. Say, $c_{k}(s) \leq 0$ for $k=k_{1}, k_{2}, \cdots, k_{p}$. Without loss of generality, we can assume

$$
X=\sum_{i=1}^{p} x_{i} \nabla c_{k_{i}}(s)
$$

We want to find $X$ such that

$$
\left\langle\nabla c_{k_{i}}(S), X\right\rangle=d c_{k_{i}}(S) X>0, \quad i=1,2, \cdots, p,
$$

where $d c_{k_{i}}(s)=\left(\nabla c_{k_{i}}(s)\right)^{T}$.

We construct a $p \times n^{2}$ matrix $G$ in the following way:

Step 1: Index the columns of $G$ by $\Lambda$ in natural order.

Step 2: Set

$$
G_{l,(i j)}=\left\{\begin{array}{l}
\frac{1}{k_{l}} G_{i j}^{k_{l}}, \quad i, j \leq k_{l} \\
0, \quad \text { otherwise; } \quad l=1,2, \cdots, p
\end{array}\right.
$$

Note that if $k=1$ then $t_{i i}$ has no cofactor in $T^{1}$. We define $G_{11}^{1}=1$. (It is consistent with the meaning of cofactors when calculating the determinant.) Then we have the following:

\section{LEMMA 6.2.}

1. Under assumption A1, for any $1 \leq k \leq n, G^{k} F x \neq 0$ for almost all $s$.

2.

$$
\left(\begin{array}{c}
<\nabla c_{k_{1}}(s), X> \\
\cdots \\
<\nabla c_{k_{p}}(s), X>
\end{array}\right)=G F F^{T} G^{T}\left(\begin{array}{c}
x_{1} \\
\cdots \\
x_{p}
\end{array}\right)
$$


3. $G F F^{T} G^{T}(s)$ is invertible for almost all $s$.

Proof. 1. Using (6.1) and the structure of $G$, one sees that

$$
\operatorname{det}\left(T^{k}\right)(S)=G^{k} F s, \quad k=1,2, \cdots, n .
$$

By assumption A1, $\left\{s \mid G^{k}(s) F=0\right\}$ is an algebraic zero set.

2. From (6.5)

$$
\left(\begin{array}{c}
d c_{k_{1}}(s) \\
\cdots \\
d c_{k_{p}}(s)
\end{array}\right)=G F
$$

(6.7) follows.

3. It suffices to show that when $p=n$ the rows of $G(s) F$ are linearly independent for almost all $S$. Assume

$$
\sum_{i=1}^{n} \lambda_{i} G^{i}(s) F=0 .
$$

Right-multiply both sides by $s$, we have

$$
\sum_{i=1}^{n} \lambda_{i} c_{i}(s)=0 .
$$

Note that $c_{i}(S)$ is a homogeneous polynomial of degree $i$, So the above non-zero polynomial can have only an algebraic zero set.

The following lemma shows that the search remains in $S_{n}$.

Lemma 6.3. $V^{-1}(X) \in S_{n}$, where $X$, defined by (6.6), is the searching direction. Proof. According to (6.5)-(6.6), it is enough to show that

$$
V^{-1}\left(F^{T}\left(G^{k}\right)^{T}\right) \in S_{n}, \quad \text { for all } k .
$$

Since $G^{k}$ consists of cofactors of a symmetric matrix, it is easy to see that $V^{-1}\left(\left(G^{k}\right)^{T}\right)$ $\in S_{n}$. Next

$$
F^{T}=I+\left(L_{C}\right)^{-T}\left(L_{A}\right)^{T}=I+\left(L_{C^{T}}\right)^{-1} L_{A^{T}}
$$

According to this form, to see that $F^{T}$ maps $S_{n}$ to $S_{n}$, we have only to show that $\left(L_{C^{T}}\right)^{-1}$ maps $S_{n}$ to $S_{n}$. It is guaranteed by the fact that both the symmetric set, $S_{n}$, and the skew symmetric set, $K_{n}$, are invariant subspaces of $L_{M}$ for any $n \times n$ matrix $M$. Hence

$$
X=F^{T} C^{T}\left(\begin{array}{c}
x_{k_{1}} \\
\cdots \\
x_{k_{p}}
\end{array}\right) \in V\left(S_{n}\right) \text {. }
$$

Summarizing Lemmas 6.1, 6.2 and 6.3, we have 
ThEOREM 6.4. Assume a point $S$ is such that $c_{k_{i}}(s) \leq 0, i=1, \cdots, p$. Under assumption $A 1$ a feasible direction $X$, which makes

$$
<\nabla c_{k_{i}}(s), X>>0, \quad i=1,2, \cdots, p
$$

exists everywhere except an algebraic zero set.

Based on the above discussion, we propose the following algorithm for searching a suitable $S$.

Algorithm 6.5.

Step 1: Set $S_{0}=I$;

Step $j(j \geq 1)$ : Check if $T^{j}\left(S_{j}\right)=F\left(S_{j}\right)>0$. If the answer is "yes" we are done. Otherwise, say $k=k_{1}, \cdots, k_{p}$ are bad set, find a feasible direction by solving the following

$$
G F F^{T} G^{T} \xi=\left(\begin{array}{c}
\frac{\left|c_{k_{1}}\left(s_{j}\right)\right|}{\sum_{i=1}^{p}\left|c_{k_{i}}\left(s_{j}\right)\right|} \\
\ldots \\
\frac{\left|c_{k_{p}}\left(s_{j}\right)\right|}{\sum_{i=1}^{p}\left|c_{k_{i}}\left(s_{j}\right)\right|}
\end{array}\right):=b .
$$

For some $k$ if $c_{k}\left(s_{j}\right)=0$, we may use a small $\epsilon>0$ to replace $c_{k}\left(s_{j}\right)$. If $G F F^{T} G$ is not invertible at $s_{j}$ a small perturbation is used.

Find the step length $L>0$ such that $s_{j+1}=s_{j}+L F^{T} G^{T} \xi>0$. Check if $T^{j+1}=F\left(s_{j+1}\right)>0$.

Note that in the algorithm $\min _{k=1}^{n} \operatorname{det}\left(T^{k}(S)\right)$ is monotonically increasing. If finally we reach a step $N$ such that $\min _{k=1}^{n} \operatorname{det}\left(T^{k}\left(S_{N}\right)\right)>0$, we are done.

The algorithm can also be used for more than two matrices case. Say we have $A_{1}, \cdots, A_{m}$ and $C$, then construct $G_{i}$ from $A_{i}$, get $b_{i}$ by (6.9), and then we just need to solve

$$
\left(\begin{array}{c}
G_{1} F_{1} \\
\cdots \\
G_{m} F_{m}
\end{array}\right)\left(F_{1}^{T} G_{1}^{T} \cdots F_{m}^{T} G_{m}^{T}\right) \xi=\left(\begin{array}{c}
b_{1} \\
\cdots \\
b_{m}
\end{array}\right) .
$$

Finally, we produce $s_{j+1}=s_{j}+L\left(F_{1}^{T} G_{1}^{T} \cdots F_{m}^{T} G_{m}^{T}\right) \xi$.

Now we cannot assure the genetic exitance of $\xi$. But we still can use the algorithm, as long as a solution is obtained.

We give a detailed iteration description for the algorithm in the following example.

Example 6.6. Consider the following two matrices $A$ and $B$

$$
A=\left(\begin{array}{ccc}
1.48 & 0 & 1 \\
1 & 3 & 2 \\
1 & 0 & 0.7
\end{array}\right), \quad B=\left(\begin{array}{ccc}
1.5 & 1 & 0 \\
0 & 1 & 2 \\
0 & 0 & 1
\end{array}\right)
$$

Choose $C=B$, set $D=A-B$ and step length $L=0.05$, and denote $c_{\min }=$ $\min _{1 \leq r \leq n} c_{r}(s)$. Then 
Time 1: $c_{\min }=-0.089221$

$$
S_{1}=\left(\begin{array}{ccc}
1.011334 & 0.015526 & -0.013654 \\
0.015526 & 0.986942 & -0.019236 \\
-0.013654 & -0.019236 & 0.991166
\end{array}\right)
$$

Time 2: $c_{\min }=-0.044875$

$$
S_{2}=\left(\begin{array}{ccc}
1.021876 & 0.037655 & -0.030578 \\
0.037655 & 0.9683 & -0.043399 \\
-0.030578 & -0.043399 & 0.988218
\end{array}\right)
$$

Time 3: $c_{\min }=-0.001875$

$$
S_{3}=\left(\begin{array}{ccc}
1.026091 & 0.070601 & -0.050337 \\
0.070601 & 0.941036 & -0.071702 \\
-0.050337 & -0.071702 & 1.003722
\end{array}\right)
$$

Time 4: $c_{m i n}=10^{-6}>0$. Now we are done. The solution is

$$
S_{4}=\left(\begin{array}{ccc}
1.026091 & 0.070601 & -0.050337 \\
0.070601 & 0.941036 & -0.071702 \\
-0.050337 & -0.071702 & 1.003722
\end{array}\right)
$$

The common quadratic Lyapunov function is determined by

$$
P=\left(L_{C}\right)^{-1}\left(S_{4}\right)=\left(\begin{array}{ccc}
0.839428 & -0.746097 & 0.19495 \\
-0.746097 & 1.545942 & -0.537712 \\
0.19495 & -0.537712 & 0.501861
\end{array}\right)
$$

EXAMPLE 6.7. Consider the following three matrices:

$$
\begin{gathered}
A_{1}=\left(\begin{array}{ccc}
2.3 & 0 & 0.8 \\
1 & 3 & 2 \\
1 & 0 & 0.7
\end{array}\right), A_{2}=\left(\begin{array}{ccc}
1.7 & 1 & 1 \\
0.8 & 2.6 & 2.3 \\
1.9 & 1.4 & 1.4
\end{array}\right), \\
A_{3}=\left(\begin{array}{ccc}
1.9 & 1 & 0 \\
0 & 1 & 2 \\
0 & 0 & 1
\end{array}\right) .
\end{gathered}
$$

We choose $C=A_{3}$ and use above algorithm with $S_{0}=I$ and step length $L=0.05$. The following is the minimum values, $c_{\min }$, of $\operatorname{det}\left(T^{k}\right)$ in seven iterations:

$c_{1}=-0.241598, c_{2}=-0.19726, c_{3}=-0.153845, c_{4}=-0.109215, c_{5}=-0.06367$, $c_{6}=-0.019166, c_{7}=10^{-6}>0$. 
After 7 iterations we are done. The solution is

$$
S_{7}=\left(\begin{array}{ccc}
0.563234 & 0.201341 & 0.0629 \\
0.201341 & 0.805909 & -0.173564 \\
0.0629 & -0.173564 & 1.088162
\end{array}\right)
$$

Then a common quadratic Lyapunov function is determined by the following $P>0$

$$
P=\left(L_{C}\right)^{-1}\left(S_{7}\right)=\left(\begin{array}{ccc}
0.500633 & -0.669585 & 0.239229 \\
-0.66958 & 1.664681 & -0.630863 \\
0.239229 & -0.630863 & 0.544081
\end{array}\right)
$$

7. Conclusion. In this paper certain properties of the Lyapunov mapping were investigated. Particularly, the spectrum decomposition and the norm are investigated. Some sufficient conditions for two matrices to share a common quadratic Lyapunov function were obtained.

The main effort is put on developing an numerical method for finding a common quadratic Lyapunov function for a set of anti-stable matrices. We first express a necessary and sufficient condition for the existence of such Lyapunov function by using the Lyapunov mappings. An estimation of the norm of the Lyapunov mappings was obtained to support the choice of a base matrix from the set. Certain properties were obtained for formulating the algorithm. A computer software was created to realize the algorithm. Two numerical examples, produced by the software, were presented to support the algorithm.

\section{References}

[1] T. OOBA AND Y. FUNAHASHI, Two conditions concerning common quadratic Lyapunov functions for linear systems, IEEE Trans. Automat. Contr., 42:5(1997), pp. 719-721.

[2] T. Ooba And Y. Funahashi, Stability robustness for linear state space models - a Lyapunov mapping approach, Sys. Contr. Lett, 29(1997), pp. 191-196.

[3] K. S. NARENDRA AND J. BALAKRISHnAN, A common Lyapunov function for stable LTI systems with commuting A-matrices, IEEE Trans. Automat. Contr., 39:12(1994), pp. 2469-2471.

[4]A. TESi And A. ViCito, Robust stability of state-space models with structures uncertainties, IEEE Trans. Aut. Contr., 35:2(1990), pp. 191-195.

[5] K. Zhou AND P. KHARgoneKar, Stability robustness for linear state-space model with structured uncertainty, IEEE Trans. Aut. Contr., 32:7(1987), pp. 621-623.

[6] K. Zhou, P. P. Khargonekar, H. Stoustrup, and H. N. Niemann, Robust stability and performance of uncertain systems in state space, Automatica, $31: 2(1995)$, pp. 249-255 
[7] M.A. Rotea, M. Corless, D. Da, And L. R. Petersen, Systems with structured uncertainty: Relations between quadratic and robust stability, IEEE Trans. Aut. Contr., 38:5(1993), pp. 779-803.

[8] P. P. Khargonekar, I. R. Petersen, and K. Zhou, Robust stabilization of uncertain linear systems: Quadratic stabilization and $H_{\infty}$ control theory, IEEE Trans. Aut. Contr., 35(1990), pp. 356-361.

[9] M.S. BRANICKY, Multiple Lyapunov functions and other analysis tools for switched and hybrid systems, IEEE Trans. Aut. Contr., 43:4(1998), pp. 475-482.

[10] Z. Sun AND D. Zheng, On Reachability and stabilization of switched linear systems, IEEE Trans. Aut. Contr., 46:2(2001), pp. 291-295.

[11] W. M. Воотнвy, An Introduction to Differentiable Manifolds and Riemannian Geometry, 2nd ed., Academic Press, 1986

[12] R. A. Abraham and J.E. Marsden, Foundations of Mechanics, 2nd Ed. Benjamin/Cummings Pub. Com. Inc., 1978.

[13] R. Bellman, Introduction to Matrix Analysis, McGraw-Hill Book Comp. 2nd ed., 1970.

[14] R.V. PATEL AND M. Toda, Quantitative measures of robustness for multivariable systems, Proc. Joint Automat. Contr. Conf., San Francisco, CA, 1980, Paper TP8-A

[15] G. Hewer And C. Kenney, The sensitivity of the stable Lyapunov equation, SIAM J. Contr. Opt., 26:2(1988), pp. 321-344. 
